\title{
Distribution of Lymph Node Metastases in Esophageal Carcinoma Patients Undergoing Upfront Surgery: A Systematic Review
}

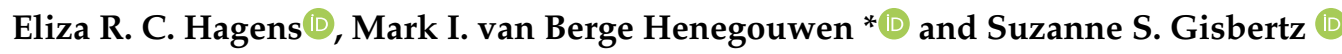 \\ Department of Surgery, Amsterdam University Medical Centers, University of Amsterdam, Cancer Center \\ Amsterdam, 1105AZ Amsterdam, The Netherlands; e.r.hagens@amsterdamumc.nl (E.R.C.H.); \\ s.s.gisbertz@amsterdamumc.nl (S.S.G.) \\ * Correspondence: m.i.vanbergehenegouwen@amsterdamumc.nl
}

Received: 25 May 2020; Accepted: 12 June 2020; Published: 16 June 2020

\begin{abstract}
Metastatic lymphatic mapping in esophageal cancer is important to determine the optimal extent of the radiation field in case of neoadjuvant chemoradiotherapy and lymphadenectomy when esophagectomy is indicated. The objective of this review is to identify the distribution pattern of metastatic lymphatic spread in relation to histology, tumor location, and T-stage in patients with esophageal cancer. Embase and Medline databases were searched by two independent researchers. Studies were included if published before July 2019 and if a transthoracic esophagectomy with a complete 2- or 3-field lymphadenectomy was performed without neoadjuvant therapy. The prevalence of lymph node metastases was described per histologic subtype and primary tumor location. Fourteen studies were included in this review with a total of 8952 patients. We found that both squamous cell carcinoma and adenocarcinoma metastasize to cervical, thoracic, and abdominal lymph node stations, regardless of the primary tumor location. In patients with an upper, middle, and lower thoracic squamous cell carcinoma, the lymph nodes along the right recurrent nerve are often affected (34\%, 24\% and $10 \%$, respectively). Few studies describe the metastatic pattern of adenocarcinoma. The current literature is heterogeneous in the classification and reporting of lymph node metastases. This complicates evidence-based strategies in neoadjuvant and surgical treatment.
\end{abstract}

Keywords: esophageal cancer; esophagectomy; lymphadenectomy; lymph node metastases; systematic review

\section{Introduction}

Esophageal cancer patients often present with an advanced disease stage, encompassing metastatic lymph nodes or distant metastases. The presence and number of lymph node metastases are among the most important prognostic factors in esophageal carcinoma and are independent predictors for long-term survival [1-6].

The location of metastatic lymph nodes depends on tumor histology, primary tumor location, T-stage and neo-adjuvant therapy [7]. The vessels in the dense lymphatic network surrounding the esophagus are complexly aligned and they contribute to a multidirectional spread of lymph node metastases in the abdomen, the mediastinum, and the neck [8,9]. Additionally, 'skip metastasis', skipping the first and directly metastasizing into the second or third lymph node echelons, are frequently seen in both esophageal adenocarcinoma and squamous cell carcinoma $[10,11]$. This contributes to the presence of lymph node metastases at unexpected distant sites, which makes standardization of the extent of the radiation field and lymphadenectomy in the treatment of esophageal cancer difficult. Not surprisingly, the optimal extent of the lymphadenectomy in esophagectomy has been subject of a global 
debate over the past decades [12,13]. In addition, several classification systems exist, contributing to the heterogeneity in the reporting of studies and clinical practice [14,15].

An extensive lymphadenectomy may result in more post-operative complications, while an insufficient lymphadenectomy carries the risk of understaging and undertreating patients, which may reduce long-term survival [16]. In Western countries, a two-field lymphadenectomy is preferred for distal esophageal adenocarcinomas. Especially in the upper mediastinum, the extent varies considerably among surgeons, centers, and countries [17]. In Asia, where predominantly squamous cell carcinoma is seen, an extensive three-field lymphadenectomy is common practice [12].

Current studies on metastatic lymphatic mapping in esophageal cancer suggest possible dissemination patterns but do not come forward with sufficient evidence to determine the optimal extent of the radiation field and lymphadenectomy. The lack of homogeneity concerning the classification of lymph node stations makes the interpretation of studies difficult and data hard to compare. In other types of cancer, such as pancreatic, breast, and in colon cancer, the standardization of lymphadenectomy has already been established and it has improved oncologic outcome in the long term [18-20].

The objective of this review is to identify the locoregional distribution of lymph node metastases in esophageal cancer patients in potentially curable patients, stratified for histology, tumor location, and T-stage. An outline on metastatic lymphatic distribution patterns may contribute to a uniform worldwide staging system and to the standardization of the extent of the radiation field and lymphadenectomy in esophageal carcinoma.

\section{Methods}

A review protocol was developed based on the Preferred Reporting Items for Systematic Reviews and Meta-Analysis (PRISMA) statement (www.prisma-statement.org) and was registered in the International Prospective Register of Systematic Reviews (PROSPERO) database (CRD42018102804). PubMed and Embase were searched on 22 July 2019. The following terms were used (including synonyms and closely related words) as index terms or free-text words: 'esophageal cancer (including junctional carcinomas)' and 'lymph node metastasis' and 'lymphadenectomy'. The full search strategies for PubMed and Embase.com can be found in Appendix A. Duplicate articles were excluded.

Articles were screened by two independent researchers (EH, SSG) in two stages: screening of titles and abstracts followed by the retrieval and screening of full-text articles. Inclusion criteria were as follows: studies describing a complete 2- or 3-field lymphadenectomy by transthoracic esophagectomy, the prevalence of patients with lymph node metastases per lymph node station is given or can be calculated, data is separately reported for adenocarcinoma and/or squamous cell carcinoma and tumor location. We excluded studies describing surgery following neo-adjuvant therapy (because the distribution may be different after neo-adjuvant chemo(radio)therapy), imaging studies, case reports, conference abstracts and reviews, and papers in another language than English or Dutch.

\subsection{Data Extraction}

The primary endpoint is the metastatic rate of lymph node metastases in esophageal carcinoma per lymph node station or region. When available, the following variables were extracted from the included studies: year of publication; country; study design; inclusion period; lymph node classification system used (JES, Japan Esophageal Society; AJCC, American Joint Committee on Cancer; other; none); description of how detailed lymph node regions or stations are described and reported; number of patients; patient characteristics (gender, age); tumor histology; tumor location (upper thoracic esophagus, middle thoracic esophagus, lower thoracic esophagus or gastroesophageal junction (GEJ)); c/pT-stage; c/pN-stage; type of surgical approach; lymphadenectomy (complete 2- or 3- field); use of immunohistochemistry staining; prevalence of lymph node metastases per lymph node station; number of patients with lymph node metastases; overall percentage of positive lymph nodes; number of patients per lymph node location with resected nodes and positive nodes in that station or region. 
Methodological quality was assessed using the Methodological Index for Non-Randomized Studies (MINORS) checklist [21].

\subsection{Statistical Analysis}

Descriptive statistics summarized the characteristics of included studies, patient characteristics, and the outcomes of each included study. Since not all studies used the same classification or definition for lymph node stations, and some studies only reported lymph node data for different regions instead of stations, we combined the two mostly used systems (JES and AJCC) and grouped lymph node stations into five regions: cervical, upper mediastinal, middle mediastinal, lower mediastinal, and abdominal (Table 1). Studies with a reported number of patients with metastatic lymph nodes per station were pooled separately from the studies only describing data per region. The prevalence of patients with lymph node metastases per station or region were calculated by summarizing all the patients with lymph node metastases per lymph node station or region and dividing them by the sum of all patients who had a lymph node dissection in this station or region. Results were stratified for tumor histology and primary tumor location. Finally, studies describing the lymphatic distribution pattern according to the pT-stage were pooled and described separately.

\section{Results}

Details of the literature search and study selection are shown in Figure 1. Fourteen studies met the inclusion criteria and were included in this review [7,22-34]. Characteristics of the included studies are presented in Table 2. A total of 8952 patients were evaluated, including $409(5 \%)$ with an adenocarcinoma and $8543(95 \%)$ with a squamous cell carcinoma. Among all patients with a squamous cell carcinoma, $726(9 \%)$ patients had a tumor located in the upper thoracic esophagus, $5130(60 \%)$ patients had a tumor in the middle thoracic esophagus and $2687(31 \%)$ had a tumor in the lower thoracic esophagus. None of these studies described patients with a cervical or GEJ squamous cell carcinoma. For adenocarcinoma, $32(8 \%)$ tumors were located in the distal esophagus and $377(92 \%)$ were located at the GEJ. The c/pT stage varied among studies, but most of the patients had a c/pT3 tumor. Details of the study populations can be found in Tables 2 and 3 . 
Table 1. Combination of JES and AJCC stations and five lymph node regions.

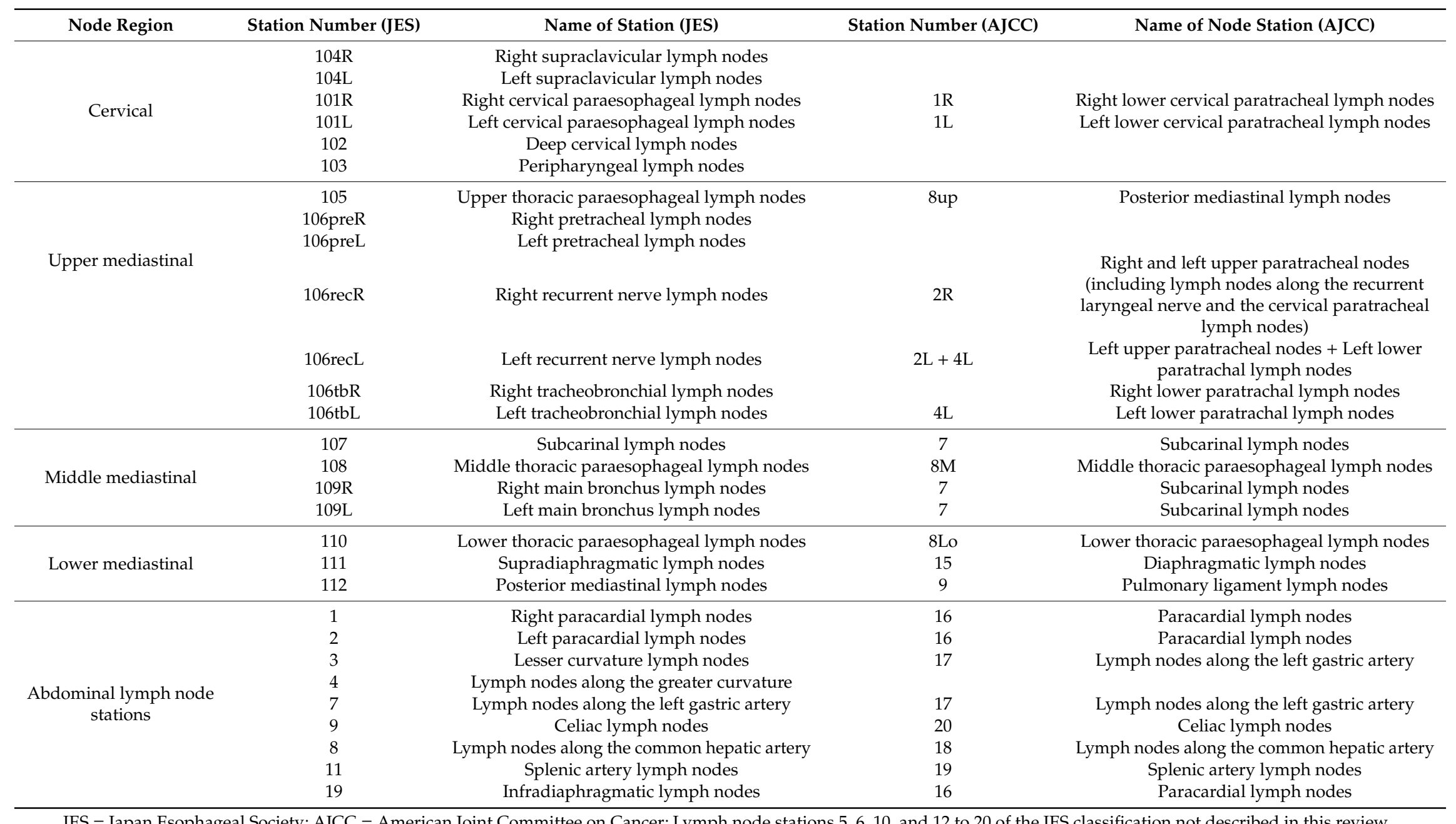

JES = Japan Esophageal Society; AJCC = American Joint Committee on Cancer; Lymph node stations 5, 6, 10, and 12 to 20 of the JES classification not described in this review. 

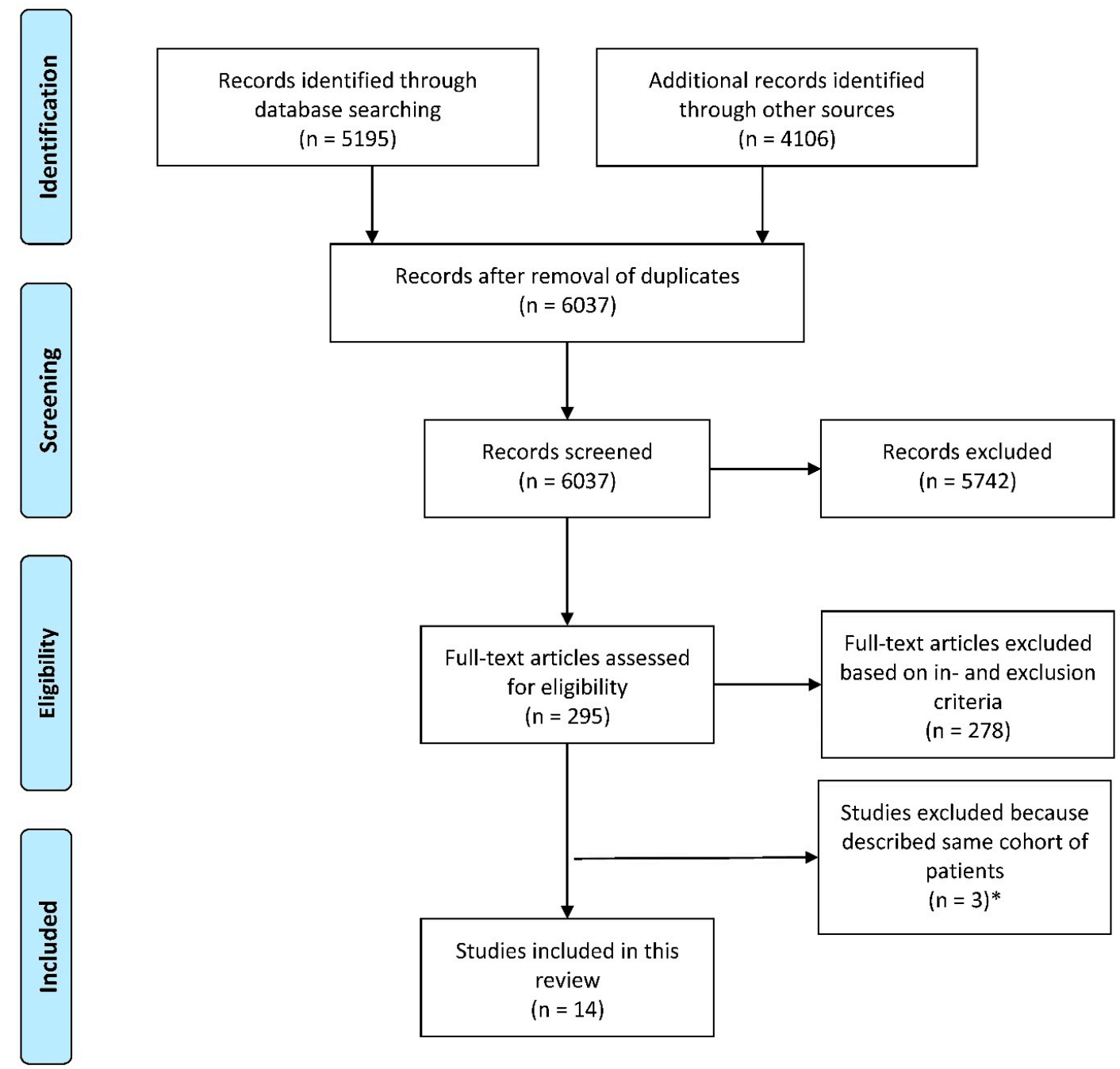

Figure 1. Flow-chart of study selection. * Three studies by Chen and colleagues and two by Li and colleagues described (partly) the same cohort of patients. Therefore, only one study of Chen et al. and one study of Li et al. were included. For both, this was the study where most of the lymph node stations and/or regions were described. 
Table 2. Characteristics of included studies.

\begin{tabular}{|c|c|c|c|c|c|c|c|c|c|c|c|c|}
\hline No & $\begin{array}{c}\text { First } \\
\text { Author }\end{array}$ & $\begin{array}{c}\text { Year of } \\
\text { Publication }\end{array}$ & $\begin{array}{c}\text { Study } \\
\text { Design }\end{array}$ & Country & $\begin{array}{l}\text { Number of } \\
\text { Patients }\end{array}$ & $\begin{array}{c}\text { Inclusion } \\
\text { Period }\end{array}$ & $\begin{array}{c}\text { 2- or 3-Field } \\
\text { Lymphadenectomy }\end{array}$ & $\begin{array}{l}\text { Lymph Node } \\
\text { Classification } \\
\text { System Used }\end{array}$ & $\begin{array}{c}\text { Use of } \\
\begin{array}{c}\text { Immunohistochemistry } \\
\text { Staining }\end{array}\end{array}$ & $\begin{array}{l}\text { MINORS } \\
\text { Score }\end{array}$ & $\begin{array}{l}\text { How Detailed Are the } \\
\text { Locations of Lymph } \\
\text { Node Metastases } \\
\text { Described? }\end{array}$ & $\begin{array}{c}\text { How Are the } \\
\text { Locations of } \\
\text { Nodal Metastases } \\
\text { Reported? }\end{array}$ \\
\hline 1 & $\begin{array}{l}\text { S. Sharma } \\
\text { [22] }\end{array}$ & 1994 & $\begin{array}{l}\text { Retrospective } \\
\text { study }\end{array}$ & Japan & 70 & 1985-1991 & 3 -field & $\begin{array}{l}\text { No standard } \\
\text { classification } \\
\text { used }\end{array}$ & NR & 10 & $\begin{array}{l}\text { Description of several } \\
\text { stations in cervical, } \\
\text { thoracic, and } \\
\text { abdominal regions. }\end{array}$ & $\begin{array}{c}\text { Number of patients } \\
\text { with resected and } \\
\text { positive lymph } \\
\text { nodes reported per } \\
\text { station. }\end{array}$ \\
\hline 2 & $\begin{array}{l}\text { C. van de } \\
\text { Ven [23] }\end{array}$ & 1999 & $\begin{array}{l}\text { Prospective } \\
\text { observational } \\
\text { study }\end{array}$ & Belgium & 37 & 1994-1998 & 3 -field & $\begin{array}{l}\text { No standard } \\
\text { classification } \\
\text { used }\end{array}$ & NR & 11 & $\begin{array}{l}\text { Description of several } \\
\text { stations in cervical, } \\
\text { thoracic, and } \\
\text { abdominal regions. }\end{array}$ & $\begin{array}{c}\text { Number of patients } \\
\text { with resected and } \\
\text { positive lymph } \\
\text { nodes reported per } \\
\text { region. }\end{array}$ \\
\hline 3 & $\begin{array}{l}\text { H. Igaki } \\
\text { [24] }\end{array}$ & 2001 & $\begin{array}{l}\text { Retrospective } \\
\text { study }\end{array}$ & Japan & 96 & 1986-1998 & 3 -field & $\begin{array}{l}\text { No standard } \\
\text { classification } \\
\text { used }\end{array}$ & NR & 8 & $\begin{array}{l}\text { No description of } \\
\text { stations. Cervical, } \\
\text { upper mediastinal, } \\
\text { middle mediastinal, } \\
\text { lower mediastinal, } \\
\text { perigastric, and celiac } \\
\text { regions described. }\end{array}$ & $\begin{array}{c}\text { Number of patients } \\
\text { with resected and } \\
\text { positive lymph } \\
\text { nodes reported per } \\
\text { region. Numbers } \\
\text { per station were } \\
\text { not provided. }\end{array}$ \\
\hline 4 & $\begin{array}{l}\text { S.M. } \\
\text { Dresner } \\
{[25]}\end{array}$ & 2001 & $\begin{array}{l}\text { Retrospective } \\
\text { study }\end{array}$ & $\begin{array}{c}\text { United } \\
\text { Kingdom }\end{array}$ & 104 & 1996-1999 & 2 -field & $\begin{array}{l}\text { No standard } \\
\text { classification } \\
\text { used }\end{array}$ & NR & 10 & $\begin{array}{l}\text { Description of stations } \\
\text { in lower thoracic and } \\
\text { abdominal regions. }\end{array}$ & $\begin{array}{c}\text { Number of patients } \\
\text { with resected and } \\
\text { positive lymph } \\
\text { nodes reported for } \\
\text { the abdominal } \\
\text { region. Numbers } \\
\text { per station were } \\
\text { not provided. }\end{array}$ \\
\hline 5 & $\begin{array}{l}\text { J. Chen } \\
{[26]}\end{array}$ & 2009 & $\begin{array}{l}\text { Retrospective } \\
\text { study }\end{array}$ & China & 1850 & 1993-2006 & 3 -field & JES & $\mathrm{H} \& \mathrm{E}$ & 12 & $\begin{array}{l}\text { Description of stations } \\
\text { according to JES in } \\
\text { cervical, upper } \\
\text { mediational, middle } \\
\text { mediastinal, lower } \\
\text { mediastinal and } \\
\text { abdominal region. }\end{array}$ & $\begin{array}{l}\text { Number of patients } \\
\text { with resected and } \\
\text { positive lymph } \\
\text { nodes reported per } \\
\text { region and station. }\end{array}$ \\
\hline 6 & $\begin{array}{c}\text { Y. } \\
\text { Tachimori } \\
\text { [27] }\end{array}$ & 2011 & $\begin{array}{l}\text { Retrospective } \\
\text { study }\end{array}$ & Japan & 356 & $2001-2005$ & 3 -field & $\begin{array}{l}\text { No standard } \\
\text { classification } \\
\text { used }\end{array}$ & NR & 10 & $\begin{array}{l}\text { No description of } \\
\text { stations. Cervical, } \\
\text { upper mediastinal, } \\
\text { middle mediastinal, } \\
\text { lower mediastinal, } \\
\text { perigastric and celiac } \\
\text { regions described. }\end{array}$ & $\begin{array}{c}\text { Number of patient } \\
\text { with resected and } \\
\text { positive lymph } \\
\text { nodes reported per } \\
\text { region. Numbers } \\
\text { per station were } \\
\text { not provided. }\end{array}$ \\
\hline
\end{tabular}


Table 2. Cont

\begin{tabular}{|c|c|c|c|c|c|c|c|c|c|c|c|c|}
\hline No & $\begin{array}{c}\text { First } \\
\text { Author }\end{array}$ & $\begin{array}{c}\text { Year of } \\
\text { Publication }\end{array}$ & $\begin{array}{c}\text { Study } \\
\text { Design }\end{array}$ & Country & $\begin{array}{l}\text { Number of } \\
\text { Patients }\end{array}$ & $\begin{array}{c}\text { Inclusion } \\
\text { Period }\end{array}$ & $\begin{array}{l}\text { 2- or 3-Field } \\
\text { Lymphadenectomy }\end{array}$ & $\begin{array}{l}\text { Lymph Node } \\
\text { Classification } \\
\text { System Used }\end{array}$ & $\begin{array}{c}\text { Use of } \\
\text { Immunohistochemistry } \\
\text { Staining }\end{array}$ & $\begin{array}{l}\text { MINORS } \\
\text { Score }\end{array}$ & $\begin{array}{l}\text { How Detailed Are the } \\
\text { Locations of Lymph } \\
\text { Node Metastases } \\
\text { Described? }\end{array}$ & $\begin{array}{c}\text { How Are the } \\
\text { Locations of } \\
\text { Nodal Metastases } \\
\text { Reported? }\end{array}$ \\
\hline 7 & $\begin{array}{c}\text { C. Castoro } \\
{[7]}\end{array}$ & 2011 & $\begin{array}{l}\text { Retrospective } \\
\text { study }\end{array}$ & Italy & 248 & 1992-2007 & 2-field and 3-field & $\begin{array}{l}\text { No standard } \\
\text { classification } \\
\text { used }\end{array}$ & H\&E and PAS & 11 & $\begin{array}{l}\text { Description of stations } \\
\text { in cervical, thoracic } \\
\text { and abdominal regions. }\end{array}$ & $\begin{array}{c}\text { Number of patients } \\
\text { with resected and } \\
\text { positive lymph } \\
\text { nodes reported per } \\
\text { station.* }\end{array}$ \\
\hline 8 & H. Li [28] & 2012 & $\begin{array}{l}\text { Retrospective } \\
\text { study }\end{array}$ & China & 200 & 2000-2010 & 3 -field & $\begin{array}{l}\text { No standard } \\
\text { classification } \\
\text { used }\end{array}$ & $\mathrm{H} \& \mathrm{E}$ & 11 & $\begin{array}{l}\text { No description of } \\
\text { stations. Cervical, } \\
\text { mediastinal, recurrent } \\
\text { laryngeal nerve and } \\
\text { abdominal regions } \\
\text { described. }\end{array}$ & $\begin{array}{l}\text { Number of patients } \\
\text { with resected and } \\
\text { positive lymph } \\
\text { nodes reported per } \\
\text { region and the } \\
\text { recurrent laryngeal } \\
\text { nerve station. }\end{array}$ \\
\hline 9 & $\begin{array}{l}\text { S. Kosugi } \\
\text { [29] }\end{array}$ & 2013 & $\begin{array}{l}\text { Retrospective } \\
\text { study }\end{array}$ & Japan & 86 & 1992-2011 & 3 -field & JES & NR & 11 & $\begin{array}{l}\text { Description of stations } \\
\text { according to JES in } \\
\text { cervical, upper } \\
\text { mediational, middle } \\
\text { mediastinal, lower } \\
\text { mediastinal, } \\
\text { perigastric, and } \\
\text { suprapancreatic } \\
\text { regions. }\end{array}$ & $\begin{array}{c}\text { Number of patients } \\
\text { with resected and } \\
\text { positive lymph } \\
\text { nodes reported per } \\
\text { station. }\end{array}$ \\
\hline 10 & $\begin{array}{l}\text { J. Cheng } \\
\text { [30] }\end{array}$ & 2013 & $\begin{array}{l}\text { Retrospective } \\
\text { study }\end{array}$ & China & 1893 & 2003-2011 & 2-field and 3-field & JES & H\&E & 9 & $\begin{array}{l}\text { Description of stations } \\
\text { according to JES in } \\
\text { cervical, upper } \\
\text { mediational, middle } \\
\text { mediastinal, lower } \\
\text { mediastinal, and } \\
\text { abdominal region. } \\
\end{array}$ & $\begin{array}{l}\text { Number of patients } \\
\text { with resected and } \\
\text { positive lymph } \\
\text { nodes reported per } \\
\text { region and per a } \\
\text { selected number of } \\
\text { stations. }\end{array}$ \\
\hline 11 & Z. Lin [31] & 2016 & $\begin{array}{c}\text { Prospective } \\
\text { observational } \\
\text { study }\end{array}$ & China & 260 & 2009-2013 & 3-field & AJCC & H\&E & 13 & $\begin{array}{l}\text { Description of stations } \\
\text { according to AJCC in } \\
\text { the thoracic and } \\
\text { abdominal region. }\end{array}$ & $\begin{array}{c}\text { Number of patients } \\
\text { with resected and } \\
\text { positive lymph } \\
\text { nodes reported per } \\
\text { station. }\end{array}$ \\
\hline 12 & $\begin{array}{l}\text { Y. Dong } \\
\text { [32] }\end{array}$ & 2015 & $\begin{array}{l}\text { Retrospective } \\
\text { study }\end{array}$ & China & 3587 & 2000-2014 & 2-field and 3-field & JES & NR & 10 & $\begin{array}{l}\text { Description of stations } \\
\text { according to JES in the } \\
\text { cervical, upper } \\
\text { mediastinal, middle } \\
\text { mediastinal, lower } \\
\text { mediastinal, and } \\
\text { abdominal regions. }\end{array}$ & $\begin{array}{c}\text { Number of patients } \\
\text { with resected and } \\
\text { positive lymph } \\
\text { nodes reported per } \\
\text { region. Numbers } \\
\text { per station were } \\
\text { not provided.* }\end{array}$ \\
\hline
\end{tabular}


Table 2. Cont.

\begin{tabular}{|c|c|c|c|c|c|c|c|c|c|c|c|c|}
\hline No & $\begin{array}{l}\text { First } \\
\text { Author }\end{array}$ & $\begin{array}{c}\text { Year of } \\
\text { Publication }\end{array}$ & $\begin{array}{l}\text { Study } \\
\text { Design }\end{array}$ & Country & $\begin{array}{l}\text { Number of } \\
\text { Patients }\end{array}$ & $\begin{array}{c}\text { Inclusion } \\
\text { Period }\end{array}$ & $\begin{array}{l}\text { 2- or 3-Field } \\
\text { Lymphadenectomy }\end{array}$ & $\begin{array}{l}\text { Lymph Node } \\
\text { Classification } \\
\text { System Used }\end{array}$ & $\begin{array}{c}\text { Use of } \\
\text { Immunohistochemistry } \\
\text { Staining }\end{array}$ & $\begin{array}{l}\text { MINORS } \\
\text { Score }\end{array}$ & $\begin{array}{l}\text { How Detailed Are the } \\
\text { Locations of Lymph } \\
\text { Node Metastases } \\
\text { Described? }\end{array}$ & $\begin{array}{c}\text { How Are the } \\
\text { Locations of } \\
\text { Nodal Metastases } \\
\text { Reported? } \\
\end{array}$ \\
\hline 13 & $\begin{array}{c}\text { X. Duan } \\
\text { [33] }\end{array}$ & 2017 & $\begin{array}{l}\text { Retrospective } \\
\text { study }\end{array}$ & China & 136 & 2014-2016 & 2-field and 3-field & $\begin{array}{l}\text { No standard } \\
\text { classification } \\
\quad \text { used }\end{array}$ & NR & 11 & $\begin{array}{l}\text { Description of stations } \\
\text { in thoracic and } \\
\text { abdominal regions. }\end{array}$ & $\begin{array}{l}\text { Number of patients } \\
\text { with resected and } \\
\text { positive lymph } \\
\text { nodes reported per } \\
\text { station. }\end{array}$ \\
\hline 14 & $\begin{array}{l}\text { S. Park } \\
\text { [34] }\end{array}$ & 2018 & $\begin{array}{l}\text { Prospective } \\
\text { observational } \\
\text { study }\end{array}$ & Korea & 29 & 2014-2018 & 3 -field & JES & H\&E & 10 & $\begin{array}{l}\text { Description of stations } \\
\text { according to JES in the } \\
\text { cervical, upper } \\
\text { mediastinal, middle } \\
\text { mediastinal, lower } \\
\text { mediastinal, and } \\
\text { abdominal regions. }\end{array}$ & $\begin{array}{l}\text { Number of patients } \\
\text { with resected and } \\
\text { positive lymph } \\
\text { nodes reported per } \\
\text { station. }\end{array}$ \\
\hline
\end{tabular}

Studies are shown in chronological order; JES = Japan Esophageal Society; AJCC = American Joint Committee on Cancer; ${ }^{*}$ Partly combined locations of the tumor in displayed data;

$\mathrm{H} \& \mathrm{E}=$ hematoxylin \& eosin; PAS = periodic acid-Schiff; NR = not reported; MINORS score ranges from 0 to 16 for non-comparative studies with 16 being the ideal score.

Table 3. Study population characteristics.

\begin{tabular}{|c|c|c|c|c|c|c|c|c|c|c|c|}
\hline No & First Author & $\begin{array}{c}\text { Sex, Male } \\
n(\%)\end{array}$ & Age, in years & Histology & $\begin{array}{c}\text { T-Stage *, } n \\
(\%)\end{array}$ & $\begin{array}{c}\text { N-Stage } *, n \\
(\%)\end{array}$ & $\begin{array}{l}\text { Location of the } \\
\text { Tumor, } n(\%)\end{array}$ & $\begin{array}{c}\text { Surgical } \\
\text { Approach }\end{array}$ & $\begin{array}{c}\text { Number of } \\
\text { Dissected } \\
\text { Lymph Nodes } \\
\text { per Patient }\end{array}$ & $\begin{array}{l}\text { Percentage of } \\
\text { Patients with } \\
\text { Lymph Node } \\
\text { Metastases }\end{array}$ & $\begin{array}{c}\text { Overall } \\
\text { Percentage of } \\
\text { Positive } \\
\text { Lymph Nodes }\end{array}$ \\
\hline 1 & S. Sharma [22] & $62(89)$ & mean 58.5 & SCC & $\begin{array}{l}\text { pT1 } 11(16) \\
\text { pT2 } 12(17) \\
\text { pT3 } 46(65) \\
\text { pT5 } 1(2)\end{array}$ & $\begin{array}{l}\text { pN0 } 20 \text { (29) } \\
\text { pN1 } 50 \text { (71) }\end{array}$ & $\begin{array}{l}\text { UTE } 10(14) \\
\text { MTE } 37 \text { (53) } \\
\text { LTE } 23(33)\end{array}$ & $\begin{array}{c}\text { Open } \\
\text { procedures }\end{array}$ & mean 82 & $\begin{array}{l}71 \% \\
(50 / 70)\end{array}$ & $\begin{array}{c}4 \% \\
(208 / 5720)\end{array}$ \\
\hline 2 & C. van de Ven [23] & NR & NR & $\mathrm{AC}$ & cT3 & NR & $\begin{array}{l}\text { LTE } 17 \text { (46) } \\
\text { GEJ } 20 \text { (54) }\end{array}$ & $\begin{array}{c}\text { Open } \\
\text { procedures }\end{array}$ & $\begin{array}{l}\text { mean 60, } \\
\text { SD } 17\end{array}$ & NR & $\begin{array}{c}14 \% \\
(323 / 2240)\end{array}$ \\
\hline 3 & H. Igaki [24] & 85 (97) & $\begin{array}{c}\text { mean 62 } \\
\text { [range 42-86] }\end{array}$ & SCC & $\begin{array}{l}\text { pT1 } 27(28) \\
\text { pT2 } 16(16) \\
\text { pT3 } 53(56)\end{array}$ & $\begin{array}{l}\text { pN0 } 36(38) \\
\text { pN1 } 60(62)\end{array}$ & LTE & $\begin{array}{c}\text { Open } \\
\text { procedures }\end{array}$ & NR & $\begin{array}{c}66 \% \\
(63 / 96)\end{array}$ & NR \\
\hline 4 & S.M. Dresner [25] & $91(88)$ & $\begin{array}{c}\text { mean } 63 \\
\text { [range 30-78] }\end{array}$ & $\mathrm{AC}$ & NR & NR & GEJ & $\begin{array}{c}\text { Open } \\
\text { procedures }\end{array}$ & $\begin{array}{c}\text { median 22 } \\
\text { [range 11-57] }\end{array}$ & $70 \%(73 / 104)$ & $\begin{array}{c}21 \% \\
(508 / 2476)\end{array}$ \\
\hline 5 & J. Chen [26] & 1351 (73) & $\begin{array}{c}\text { median } 55 \\
\text { [range 27-54] }\end{array}$ & SCC & $\begin{array}{l}\text { cT1 } 109(6) \\
\text { cT2 } 348(19) \\
\text { cT3 } 1215(65) \\
\text { cT4 } 178(10)\end{array}$ & NR & $\begin{array}{c}\text { UTE } 289(16) \\
\text { MTE } 1381(74) \\
\text { LTE } 180 \text { (10) }\end{array}$ & $\begin{array}{c}\text { Open } \\
\text { procedures }\end{array}$ & $\begin{array}{c}\text { mean 26 } \\
\text { [range 15-71] }\end{array}$ & $\begin{array}{c}58 \% \\
(1081 / 1850)\end{array}$ & $\begin{array}{c}9 \% \\
(4350 / 47470)\end{array}$ \\
\hline
\end{tabular}


Table 3. Cont

\begin{tabular}{|c|c|c|c|c|c|c|c|c|c|c|c|}
\hline No & First Author & $\begin{array}{c}\text { Sex, Male } \\
n(\%)\end{array}$ & Age, in years & Histology & $\begin{array}{l}\text { T-Stage *, } n \\
(\%)\end{array}$ & $\begin{array}{l}\text { N-Stage *, } n \\
(\%)\end{array}$ & $\begin{array}{l}\text { Location of the } \\
\text { Tumor, } n(\%)\end{array}$ & $\begin{array}{l}\text { Surgical } \\
\text { Approach }\end{array}$ & $\begin{array}{l}\text { Number of } \\
\text { Dissected } \\
\text { Lymph Nodes } \\
\text { per Patient }\end{array}$ & $\begin{array}{l}\text { Percentage of } \\
\text { Patients with } \\
\text { Lymph Node } \\
\text { Metastases }\end{array}$ & $\begin{array}{c}\text { Overall } \\
\text { Percentage of } \\
\text { Positive } \\
\text { Lymph Nodes }\end{array}$ \\
\hline 6 & Y. Tachimori [27] & $314(88)$ & $\begin{array}{c}\text { mean 63 } \\
\text { [range 41-80] }\end{array}$ & SCC & $\begin{array}{l}\text { pT1 } 127(36) \\
\text { pT2 } 40(11) \\
\text { pT3 } 183(51) \\
\text { pT4 } 6(2)\end{array}$ & $\begin{array}{l}\text { pN0 } 110(31) \\
\text { pN1 } 116(33) \\
\text { pN2 } 81(23) \\
\text { pN3 } 49(13)\end{array}$ & $\begin{array}{l}\text { UTE } 55 \text { (15) } \\
\text { MTE } 173(49) \\
\text { LTE } 128 \text { (36) }\end{array}$ & $\begin{array}{c}\text { Open } \\
\text { procedures }\end{array}$ & NR & NR & NR \\
\hline 6 & C. Castoro [7] & $327(81)$ & $\begin{array}{c}\text { median } 63 \\
\text { [IQR 56-70] }\end{array}$ & $\begin{array}{l}\text { SCC } 116(47) \\
\text { AC } 132(53)\end{array}$ & $\begin{array}{c}\text { cT1 } 5(2) \\
\text { cT2 } 42(17) \\
\text { cT3 } 201 \text { (81) }\end{array}$ & $\begin{array}{l}\text { cN0 } 107(43) \\
\text { cN1 } 141(57)\end{array}$ & $\begin{array}{c}\text { UTE (all SCC) } 25(10) \\
\text { MTE (all SCC) } 50(20) \\
\text { LTE (AC 15, SCC 41) } \\
56(23) \\
\text { GEJ (all AC) } 117 \text { (47) }\end{array}$ & $\begin{array}{c}\text { Open } \\
\text { procedures }\end{array}$ & $\begin{array}{l}\text { AC median } 19.5 \\
\text { [IQR 15-27] } \\
\text { SCC median } 16 \\
\text { [IQR 12-21] }\end{array}$ & $\begin{array}{l}\text { AC } 54 \% \\
(63 / 116) \\
\text { SCC } 67 \% \\
(88 / 132)\end{array}$ & NR \\
\hline 8 & H. Li [28] & $163(82)$ & $\begin{array}{l}\text { mean } 57 \\
\text { SD } 9\end{array}$ & SCC & $\begin{array}{l}\text { pT1 } 18(9) \\
\text { pT2 } 45(23) \\
\text { pT3 114 (56) } \\
\text { pT4 } 23(12)\end{array}$ & NR & $\begin{array}{l}\text { UTE } 31 \text { (15) } \\
\text { MTE } 137(69) \\
\text { LTE } 32 \text { (16) }\end{array}$ & $\begin{array}{c}\text { Open } \\
\text { procedures }\end{array}$ & NR & NR & NR \\
\hline 9 & S. Kosugi [29] & $78(91)$ & $\begin{array}{l}\text { mean 60, } \\
\text { SD } 7\end{array}$ & SCC & $\begin{array}{c}\text { pT1a 7 (8) } \\
\text { pT1b } 75(87) \\
\text { pT2 } 4 \text { (5) }\end{array}$ & $\begin{array}{l}\text { pN0 } 48(56) \\
\text { pN1 } 31(36) \\
\text { pN2 } 6(7) \\
\text { pN3 } 1(1)\end{array}$ & $\begin{array}{l}\text { UTE } 17(20) \\
\text { MTE } 59(69) \\
\text { LTE } 10(11)\end{array}$ & $\begin{array}{c}\text { Open } \\
\text { procedures }\end{array}$ & NR & $\begin{array}{c}47 \% \\
(40 / 86)\end{array}$ & NR \\
\hline 10 & J. Cheng [30] & 1474 (78) & $\begin{array}{c}<40: 1 \% \\
41-59: 48 \% \\
\geq 60: 51 \%\end{array}$ & SCC & $\begin{array}{l}\text { cTis } 10(1) \\
\text { cT1 } 103(5) \\
\text { cT2 } 345(18) \\
\text { cT4 } 1173(62) \\
\text { cT4 } 262(14)\end{array}$ & NR & $\begin{array}{l}\text { UTE } 82(4) \\
\text { MTE } 1266 \text { (67) } \\
\text { LTE } 545 \text { (29) }\end{array}$ & $\begin{array}{c}\text { Open } \\
\text { procedures }\end{array}$ & mean 13 & $\begin{array}{c}46 \% \\
(865 / 1893)\end{array}$ & NR \\
\hline 11 & Z. Lin [31] & $59(23)$ & $\begin{array}{l}\text { median } 61 \\
\text { [IQR 52-67] }\end{array}$ & SCC & $\begin{array}{l}\text { pT1 } 30(11) \\
\text { pT2 } 44(17) \\
\text { pT3 164 (63) } \\
\text { pT4 } 22(9)\end{array}$ & $\begin{array}{l}\text { pN0 } 119(46) \\
\text { pN1 67 (25) } \\
\text { pN2 } 54(21) \\
\text { pN3 } 20(8)\end{array}$ & $\begin{array}{l}\text { UTE } 28(11) \\
\text { MTE } 173(67) \\
\text { LTE } 59(22)\end{array}$ & $\begin{array}{c}\text { Open } \\
\text { procedures }\end{array}$ & $\begin{array}{l}\text { median } 35 \\
\text { [IQR 25-46] }\end{array}$ & $\begin{array}{c}54 \% \\
(141 / 260)\end{array}$ & $\begin{array}{c}15 \% \\
(316 / 2097)\end{array}$ \\
\hline 12 & Y. Dong [32] & $2536(72)$ & median 61 & SCC & $\begin{array}{l}\text { pT1 } 435(14) \\
\text { pT2 } 935(25) \\
\text { pT3 } 1992(55) \\
\text { pT4 225 (6) }\end{array}$ & $\begin{array}{l}\text { pN0 } 2223(62) \\
\text { pN1 } 1233(34) \\
\text { pN2 } 98(3) \\
\text { pN3 } 33(1)\end{array}$ & $\begin{array}{l}\text { UTE } 189 \text { (5) } \\
\text { MTE } 1837 \text { (51) } \\
\text { LTE } 1561 \text { (44) }\end{array}$ & $\begin{array}{l}\text { Hybrid } \\
\text { procedures }\end{array}$ & $\begin{array}{c}\text { mean 20 } \\
\text { [range 16-50] }\end{array}$ & $\begin{array}{c}38 \% \\
(1.364 / 3587)\end{array}$ & $\begin{array}{c}4 \% \\
(2870 / 71740)\end{array}$ \\
\hline 13 & X. Duan [33] & $128(95)$ & $\begin{array}{l}\text { mean } 63, \\
\text { SD } 9\end{array}$ & $\mathrm{AC}$ & $\begin{array}{l}\text { pT1-2 } 17 \text { (13) } \\
\text { pT3-4 } 119 \text { (87) }\end{array}$ & $\begin{array}{l}\text { pN0 } 44(32) \\
\text { pN1 } 64(47) \\
\text { pN2 } 21(15) \\
\text { pN3 } 7(6)\end{array}$ & GEJ & $\begin{array}{c}\text { Open } \\
\text { procedures }\end{array}$ & mean 15 & $\begin{array}{c}68 \% \\
(92 / 136)\end{array}$ & $\begin{array}{c}21 \% \\
(431 / 2083)\end{array}$ \\
\hline 14 & S. Park [34] & $26(90)$ & $\begin{array}{l}\text { mean 63, } \\
\text { SD 7 }\end{array}$ & SCC & cT1 & $\begin{array}{l}\text { cN0 } 25 \text { (86) } \\
\text { cN1 } 4 \text { (14) }\end{array}$ & $\begin{array}{l}\text { MTE } 17 \text { (59) } \\
\text { LTE } 12 \text { (41) }\end{array}$ & $\begin{array}{l}\text { Robot-assisted } \\
\text { procedures }\end{array}$ & $\begin{array}{l}\text { mean } 55, \\
\text { SD 17 }\end{array}$ & $\begin{array}{c}86 \% \\
(25 / 29)\end{array}$ & NR \\
\hline
\end{tabular}

Studies are shown in chronological order; $\mathrm{SCC}=$ squamous cell carcinoma; $\mathrm{AC}=$ adenocarcinoma; $\mathrm{GEJ}$ = gastroesophageal junction; $\mathrm{CE}=\mathrm{Cervical}$ esophagus; UTE = Upper thoracic esophagus; $\mathrm{MTE}=$ middle thoracic esophagus; $\mathrm{LTE}=$ lower thoracic esophagus; $\mathrm{NR}=$ not reported; $\mathrm{SD}=$ standard deviation; $\mathrm{IQR}=$ interquartile range; ${ }^{*}$ either pathological or clinical $\mathrm{T}$ and N-stage, based on what is reported in the paper. 


\subsection{Reporting Standard}

Standard reported lymphadenectomy differed among studies. In 1 study, patients underwent a 2-field lymphadenectomy, in 9 studies, patients underwent a 3-field lymphadenectomy, and in 4 studies, both procedures were included, resulting in a different nodal yield per study. In addition, the definition of anatomical locations of lymph node stations differed amongst studies; 1 study used AJCC, 5 used JES, and 8 did not use a standard classification system. Moreover, 6 studies described the prevalence of lymph node metastases per lymph node station, 5 only described the prevalence of lymph node metastases per region, and 3 reported a combination of both. The reported regions and stations also varied among studies (Table 2). Some studies described for some stations both sides together, and other studies separated left and right. One study combined tumor locations when reporting the number of patients per lymph node station and could therefore not be pooled with the others studies [7].

\subsection{Distribution Pattern for Esophageal Squamous Cell Carcinoma}

Eleven studies $[7,22,24,26-32,34]$ described the location of lymph node metastases in patients with a squamous cell carcinoma $(n=8543)$. Table 4 shows the prevalence of lymph node metastases per lymph node station among the seven studies [22,26,28-31,34] that reported data per lymph node station per tumor location. For patients with an upper thoracic tumor, lymph node metastases are most frequently seen along the right recurrent nerve $(60 \%)$ and cervical paraesophageal lymph nodes (right $34 \%$ and left $22 \%$ ). For patients with a middle thoracic tumor, the prevalence of lymph node metastases was highest along the right recurrent nerve (23\%), right cervical paraesophageal lymph nodes $(24 \%)$, and middle thoracic paraesophageal lymph nodes $(23 \%)$. The lymph nodes along the left gastric artery $(28 \%)$ and lower thoracic esophagus (23\%) had the highest prevalence of lymph node metastases in patients with a tumor in the lower thoracic esophagus. Six studies $[24,26-28,30,32]$ described the location of the lymph node metastases per region. The results of these studies are shown in Figure 2. 
Table 4. Prevalence of lymph node metastases per lymph node station.

\begin{tabular}{|c|c|c|c|c|c|c|c|c|c|c|c|c|c|c|c|c|}
\hline \multirow{4}{*}{$\begin{array}{c}\text { Lymph Node Station } \\
\text { Cervical region }\end{array}$} & \multicolumn{12}{|c|}{ Squamous Cell Carcinoma } & \multirow{2}{*}{\multicolumn{4}{|c|}{$\begin{array}{c}\text { Adenocarcinoma } \\
\text { Gastroesophageal Junction }\end{array}$}} \\
\hline & \multicolumn{4}{|c|}{ Upper Thoracic Esophagus } & \multicolumn{4}{|c|}{ Middle Thoracic Esophagus } & \multicolumn{4}{|c|}{ Lower Thoracic Esophagus } & & & & \\
\hline & & & & & & & & & & & & & & & & \\
\hline & $9 \%$ & 34 & 1 & 381 & $10 \%$ & 266 & / & 2684 & $13 \%$ & 100 & / & 748 & NR & & & \\
\hline Left supraclavicular lymph nodes & $5 \%$ & 14 & 1 & 299 & $5 \%$ & 67 & I & 1418 & $3 \%$ & 6 & 1 & 203 & NR & & & \\
\hline Right cervical paraesophageal lymph nodes & $34 \%$ & 103 & 1 & 299 & $24 \%$ & 345 & I & 1418 & $10 \%$ & 20 & 1 & 203 & NR & & & \\
\hline Left cervical paraesophageal lymph nodes & $22 \%$ & 65 & / & 299 & $11 \%$ & 152 & i & 1418 & $4 \%$ & 8 & 1 & 203 & NR & & & \\
\hline Right deep cervical lymph nodes & $2 \%$ & 5 & I & 289 & $<1 \%$ & 4 & I & 1381 & $0 \%$ & 0 & 1 & 180 & NR & & & \\
\hline Left deep cervical lymph nodes & $2 \%$ & 5 & 1 & 289 & $1 \%$ & 8 & 1 & 1381 & $0 \%$ & 0 & 1 & 180 & NR & & & \\
\hline \multirow{2}{*}{\multicolumn{17}{|c|}{ Upper mediastinal region }} \\
\hline & & & & & & & & & & & & & & & & \\
\hline Upper thoracic paraesophageal lymph nodes & $10 \%$ & 40 & I & 388 & $6 \%$ & 163 & I & 2811 & $3 \%$ & 7 & 1 & 214 & NR & & & \\
\hline Right pretracheal lymph nodes & $12 \%$ & 43 & 1 & 371 & $6 \%$ & 81 & / & 1381 & $2 \%$ & 3 & 1 & 180 & NR & & & \\
\hline Left pretracheal lymph nodes & $9 \%$ & 28 & 1 & 299 & $7 \%$ & 101 & I & 1418 & $2 \%$ & 4 & 1 & 203 & NR & & & \\
\hline Right recurrent nerve lymph nodes & $60 \%$ & 6 & 1 & 10 & $23 \%$ & 15 & / & 66 & $15 \%$ & 8 & i & 52 & NR & & & \\
\hline Left recurrent nerve lymph nodes & $11 \%$ & 32 & I & 289 & $7 \%$ & 102 & I & 1410 & $3 \%$ & 7 & 1 & 209 & NR & & & \\
\hline Tracheobronchial lymph nodes & $12 \%$ & 10 & I & 82 & $12 \%$ & 17 & I & 145 & $6 \%$ & 3 & 1 & 49 & NR & & & \\
\hline \multicolumn{17}{|l|}{ Middle mediastinal region } \\
\hline Subcarinal lymph nodes & $8 \%$ & 32 & I & 398 & $18 \%$ & 517 & I & 2913 & $14 \%$ & 121 & I & 836 & $25 \%$ & 1 & 1 & 4 \\
\hline Middle thoracic paraesophageal lymph nodes & $5 \%$ & 20 & I & 388 & $23 \%$ & 595 & i & 2542 & $21 \%$ & 170 & 1 & 804 & $2 \%$ & 1 & 1 & 45 \\
\hline Right main bronchus lymph nodes & $<1 \%$ & 1 & I & 289 & $2 \%$ & 24 & i & 1410 & $2 \%$ & 4 & 1 & 209 & $0 \%$ & 0 & 1 & 10 \\
\hline Left main bronchus lymph nodes & $1 \%$ & 2 & i & 289 & $3 \%$ & 37 & I & 1410 & $2 \%$ & 5 & I & 209 & $6 \%$ & 2 & 1 & 31 \\
\hline \multicolumn{17}{|l|}{ Lower mediastinal region } \\
\hline Lower thoracic paraesophageal lymph nodes & $3 \%$ & 12 & I & 388 & $8 \%$ & 221 & I & 2851 & $23 \%$ & 184 & 1 & 809 & $10 \%$ & 10 & 1 & 96 \\
\hline Supradiaphragmatic lymph nodes & $0 \%$ & 0 & i & 306 & $<1 \%$ & 3 & / & 1504 & $5 \%$ & 39 & i & 778 & $0 \%$ & 0 & I & 5 \\
\hline \multirow{2}{*}{\multicolumn{17}{|c|}{ Abdominal region }} \\
\hline & & & & & & & & & & & & & & & & \\
\hline Right paracardial lymph nodes & $1 \%$ & 2 & / & 199 & $3 \%$ & 48 & / & 1447 & $12 \%$ & 27 & I & 232 & $26 \%$ & 33 & 1 & 128 \\
\hline Left paracardial lymph nodes & $3 \%$ & 10 & / & 299 & $7 \%$ & 201 & i & 2684 & $13 \%$ & 97 & 1 & 748 & $37 \%$ & 48 & i & 131 \\
\hline Lesser curvature lymph nodes & $3 \%$ & 9 & / & 299 & $10 \%$ & 273 & i & 2713 & $11 \%$ & 89 & / & 777 & $29 \%$ & 37 & i & 127 \\
\hline Lymph nodes along the greater curvature & $0 \%$ & 0 & / & 289 & $<1 \%$ & 1 & / & 1381 & $0 \%$ & 0 & / & 180 & $12 \%$ & 5 & i & 41 \\
\hline Lymph nodes along the left gastric artery & $4 \%$ & 11 & / & 299 & $16 \%$ & 238 & / & 1528 & $28 \%$ & 76 & / & 269 & $48 \%$ & 29 & l & 60 \\
\hline Celiac lymph nodes & NR & & & & $2 \%$ & 1 & / & 56 & $3 \%$ & 1 & / & 33 & $14 \%$ & 4 & i & 29 \\
\hline Lymph nodes along the common hepatic artery & $<1 \%$ & 1 & I & 289 & $3 \%$ & 40 & I & 1483 & $5 \%$ & 37 & / & 774 & $14 \%$ & 5 & l & 37 \\
\hline Splenic artery lymph nodes & NR & & & & $2 \%$ & 1 & / & 59 & $0 \%$ & 0 & 1 & 31 & $26 \%$ & 11 & 1 & 43 \\
\hline Infradiaphragmatic lymph nodes & NR & & & & NR & & & & NR & & & & NR & & & \\
\hline Subaortic lymph nodes & NR & & & & $10 \%$ & 2 & / & 21 & $0 \%$ & 0 & I & 6 & NR & & & \\
\hline Para-aortic lymph nodes & NR & & & & $10 \%$ & 1 & / & 10 & $0 \%$ & 0 & / & 3 & NR & & & \\
\hline
\end{tabular}

$\mathrm{NR}=$ not reported. Data presented as percentage of patients with lymph node metastases with number of patients with (number of patients with metastatic lymph nodes/number of patients with lymph node dissection of this station). Only studies that presented data per lymph node station were included in this table. 

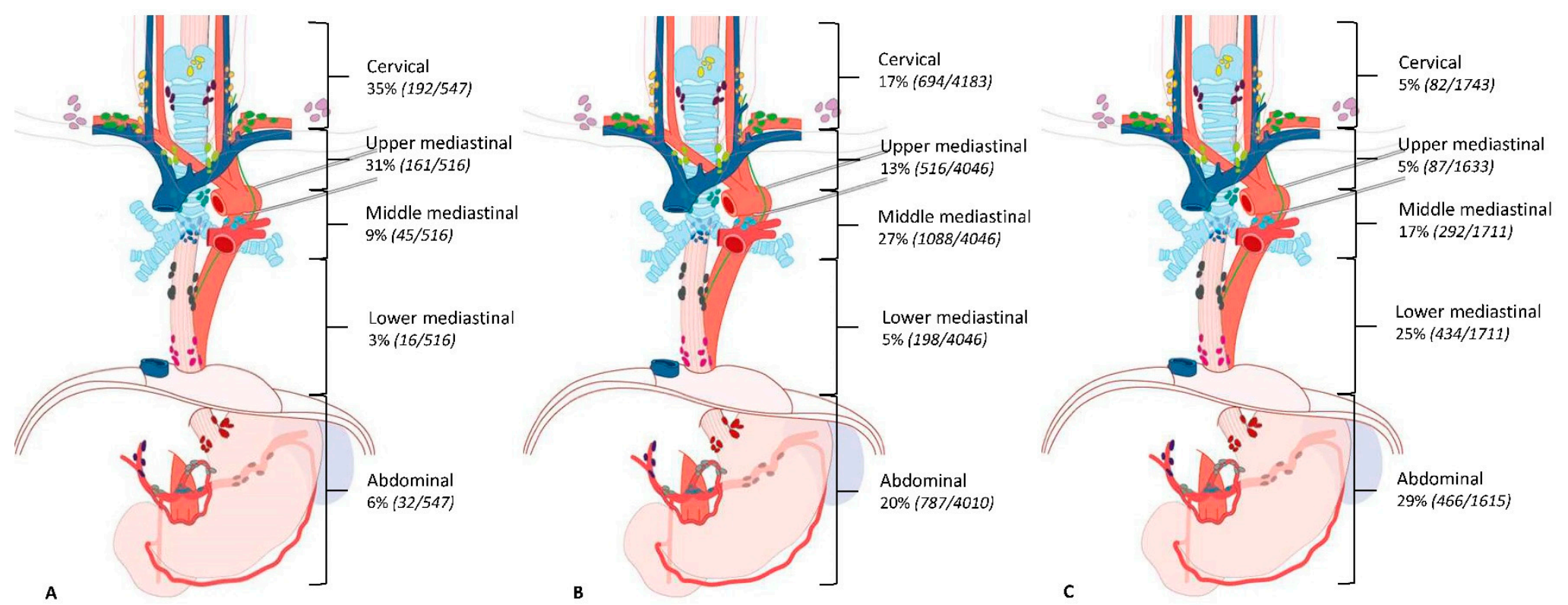

Figure 2. Prevalence of lymph node metastases per tumor location in patients with squamous cell carcinoma. $(\mathbf{A})=$ Upper thoracic esophageal tumor, $(\mathbf{B})=$ Middle thoracic esophageal tumor, $(\mathbf{C})=$ Distal thoracic esophageal tumor. Data presented as percentage of patients with lymph node metastases in this region. 


\subsection{Distribution Pattern for Esophageal Adenocarcinoma}

Four studies $[7,23,25,33]$ described the location of lymph node metastases in patients with an adenocarcinoma $(n=409)$. One study [33] described the prevalence of metastatic lymph nodes per lymph node station; this was for GEJ tumors (Table 4). Lymph node stations with the highest prevalence of patients with metastatic lymph nodes were lymph nodes along the left gastric artery (48\%), lesser curvature (29\%), splenic artery (26\%), right paracardial lymph nodes (26\%), and subcarinal lymph nodes $(25 \%)$.

Two studies $[23,25]$ described the location of lymph node metastases in regions. Pooled numbers show that for patients with a GEJ tumor, 20\% (4 out of 10) had lymph node metastases in the cervical region and $25 \%$ (31 out of 124 ) had metastases in the abdominal lymph node stations (other regions were not reported). For patients with an adenocarcinoma of the lower thoracic esophagus, 35\% (6 out of 17) had lymph node metastases in the cervical region, $71 \%$ (12 out of 17) had lymph node metastases in the lower mediastinal region, and 71\% (12 out of 17) had lymph node metastases in the abdominal region.

One study [7] combined patients with a tumor of the distal esophagus and GEJ. In this study, a prevalence of $30 \%$ in the periesophageal lymph nodes, 37\% in the paracardial lymph nodes, 35\% in the perigastric lymph nodes, and $14 \%$ in the celiac axis was reported.

\subsection{Distribution of LN Metastases in Relation to pT-Stage}

Three studies $[24,27,30]$ stratified the prevalence of nodal metastases per pT-stage. All three studies described patients with squamous cell carcinoma. Table 5 shows the rate of patients with lymph node metastases per region, divided into four groups: patients with pT1-2 and pT3-4 and patients with pT1 and pT2-4. Among patients with higher T-stages, a higher prevalence of lymph node metastases is seen per region, while the distribution remained similar.

Table 5. Lymph node metastases per pathological T-stage of patients with esophageal carcinoma.

\begin{tabular}{|c|c|c|c|c|c|c|c|c|c|c|}
\hline \multirow{3}{*}{ Lymph Node Region } & \multicolumn{2}{|c|}{ MTE } & \multicolumn{2}{|c|}{ LTE } & \multicolumn{2}{|c|}{ UTE } & \multicolumn{2}{|c|}{ MTE } & \multicolumn{2}{|c|}{ LTE } \\
\hline & pT1-2 & pT2-3 & pT1-2 & pT2-3 & pT1 & pT2-4 & pT1 & pT2-4 & pT1 & pT2-4 \\
\hline & $n=315$ & $n=915$ & $n=171$ & $n=470$ & $n=22$ & $n=33$ & $n=67$ & $\mathrm{n}=106$ & $n=38$ & $n=90$ \\
\hline Cervical region & $3 \%$ & $5 \%$ & $2 \%$ & $3 \%$ & $14 \%$ & $21 \%$ & $12 \%$ & $25 \%$ & $0 \%$ & $6 \%$ \\
\hline Upper mediastinal region & $3 \%$ & $6 \%$ & $2 \%$ & $5 \%$ & $55 \%$ & $85 \%$ & $22 \%$ & $61 \%$ & $13 \%$ & $27 \%$ \\
\hline Middle mediastinal region & $18 \%$ & $38 \%$ & $9 \%$ & $19 \%$ & $5 \%$ & $9 \%$ & $6 \%$ & $49 \%$ & $5 \%$ & $23 \%$ \\
\hline Lower mediastinal region & $2 \%$ & $3 \%$ & $27 \%$ & $39 \%$ & $0 \%$ & $9 \%$ & $9 \%$ & $25 \%$ & $5 \%$ & $27 \%$ \\
\hline Abdominal region & $11 \%$ & $16 \%$ & $25 \%$ & $33 \%$ & NR & NR & NR & NR & NR & NR \\
\hline Perigastric region & NR & NR & NR & NR & $0 \%$ & $6 \%$ & $24 \%$ & $54 \%$ & $39 \%$ & $66 \%$ \\
\hline Celiac region & NR & NR & NR & NR & $0 \%$ & $9 \%$ & $3 \%$ & $5 \%$ & $0 \%$ & $9 \%$ \\
\hline
\end{tabular}

Data presented as percentage of patients with lymph node metastases in this region. UTE $=$ Upper thoracic esophagus; MTE = Middle thoracic esophagus; LTE = Lower thoracic esophagus; NR = not reported.

\section{Discussion}

This study describes the sites of lymph node metastases in esophageal cancer patients according to the histology and primary tumor location based on literature published before July 2019. This is the first study systematically combining available evidence on lymph node metastases pattern, contributing toward revealing the lymphatic metastatic pattern of esophageal carcinoma. This study showed that both squamous cell carcinoma and adenocarcinoma metastasize to cervical, thoracic, and abdominal lymph node stations, regardless of the location of the primary tumor.

\subsection{Lymphatic Distribution Pattern for Squamous Cell Carcinoma}

Multiple studies have attempted to define the lymph node metastases pattern in esophageal cancer [7,22,23,25-27,30-33]. Most of these studies describe squamous cell carcinoma only. The available evidence of lymph node metastases pattern in adenocarcinoma of the esophagus is scarce, and the available literature for both tumor types is very heterogeneous. Whereas some studies report data per lymph node station, others report per region. To make this even more complex, not all studies 
adhere to the same boundaries of lymph node regions and not all studies use the same anatomical definition of lymph node stations. To define anatomical sites on lymph node stations, some use standardized classification systems such as the AJCC or JES, while others do not use any standardized classification system. Moreover, not all studies report the exact extent of lymphadenectomy. In addition, some studies excluded from this review combined patients with adenocarcinoma and squamous cell carcinoma and/or different tumor locations, which makes data hard to interpret, since these factors could influence the distribution pattern [35-37] All together, these factors make comparing available evidence on the lymph node metastases distribution in esophageal cancer difficult.

For upper, middle, as well as lower thoracic esophageal squamous cell carcinoma, the stations around the esophagus are among those with the highest prevalence of lymph node metastases. However, not only lymph node stations in the same region as the tumor have a high prevalence of nodal metastases; stations in different regions are affected as well. For example, 13\% of the patients with a lower esophageal tumor have right cervical lymph node metastases.

\subsection{Lymphatic Distribution Pattern for Adenocarcinoma}

For adenocarcinoma, although data are more limited, similar results are seen. One-quarter $(25 \%)$ of patients with a GEJ adenocarcinoma had middle thoracic paraesopahgeal lymph node metastases, and when looking at zones, $20 \%$ of the patients with a GEJ adenocarcinoma had lymph node metastases in the cervical zone. An explanation could be the presence of an extensive lymphatic network in the submucosa and even in the lamina propria of the esophagus, with both intramural and longitudinal lymphatic drainage. The longitudinal nature of this network explains the variation in anatomic sites of lymph node metastases [38-40]. Another result of the complexity of the lymphatic vessel system is the phenomenon of skip metastases [37]. Skip metastases are distant lymph nodes with metastatic involvement, without tumor infiltration in the regional lymph nodes, and they are more often seen in early tumors [10]. It is unclear what the exact clinical value is since the literature is conflicting on the prognostic relevance [41-43].

Three studies $[24,27,30]$ described lymph node metastases per lymph node station in relation to pT-stage in patients with squamous cell carcinoma. An increased prevalence of lymph node metastases is seen per region in patients with a higher pT-stage, while the distribution remained similar. These are small numbers; nevertheless, the literature points out that a higher T-stage is associated with more lymph node metastases [44].

It should be pointed out that this study defines the lymph node metastases pattern based on patients without neoadjuvant treatment because lymph node involvement may differ after neoadjuvant chemo(radio)therapy [45]. Currently, neoadjuvant chemoradiation or perioperative or neoadjuvant chemotherapy is the standard of care in most countries. This makes our results less applicable to current surgical patients, and one of the main questions for the future is whether the lymphadenectomy strategy should be based on the pattern of lymph node metastases before neoadjuvant treatment or after neoadjuvant treatment. However, the location of lymph node metastases in untreated esophageal cancer patients tells us more about the behavior of the disease, and this is fundamental, as this allows for accurately defining neoadjuvant treatment strategies by targeting high-risk regions for lymph node metastases in patients with specific characteristics. A recent study showed that after neoadjuvant chemoradiotherapy, almost half of the patients in that cohort had lymph node metastases outside the radiation field, indicating that the current radiation fields are not sufficient [46]. Although, it should be noted that radiotherapy to an elective nodal area (both metastatic and non-metastatic) does not guarantee a better outcome [47].

There are some limitations of the present study. Firstly, as previously mentioned, studies were very heterogeneous in lymph node dissection and the reporting of anatomical sites of nodal metastases. This heterogeneity might have made our pooled results less reliable. Moreover, not all studies could be pooled per station (since they only described lymph node regions) and vice versa. The inclusion of different studies in Tables 2 and 4 makes displayed percentages slightly different. In addition, few of 
the studies subdivided patients for T-stage and location of the primary tumor, whilst it has been proven that these factors influence lymph node metastases [26,48,49].

If we want to determine the exact distribution pattern of esophageal cancer, large well-designed prospective studies are needed. One initiative of such a study is the multinational prospective TIGER study (ClinicalTrials.gov Identifier: NCT03222895) [50].

\section{Conclusions}

Both esophageal squamous cell carcinoma and adenocarcinoma are aggressive diseases that can metastasize to cervical, thoracic, as well as abdominal lymph node stations, regardless of the location of the primary tumor. The prevalence of patients with metastatic lymph nodes per station and region could be determined for squamous cell carcinoma. However, few studies described the distribution of lymph node metastases for esophageal adenocarcinoma, and the data for both tumor types was very heterogeneous. This complicates evidence-based treatment strategies in both neoadjuvant (radiation field) and surgical (lymphadenectomy) treatment. Well-designed prospective studies are needed to determine the exact lymphatic distribution pattern of esophageal cancer.

Funding: This research received no external funding.

Conflicts of Interest: The authors declare no conflict of interest.

\section{Appendix A}

Table A1. Search strategy for PubMed (22 July 2019).

\begin{tabular}{|c|c|c|}
\hline Search & Query & Items Found \\
\hline$\# 1$ & 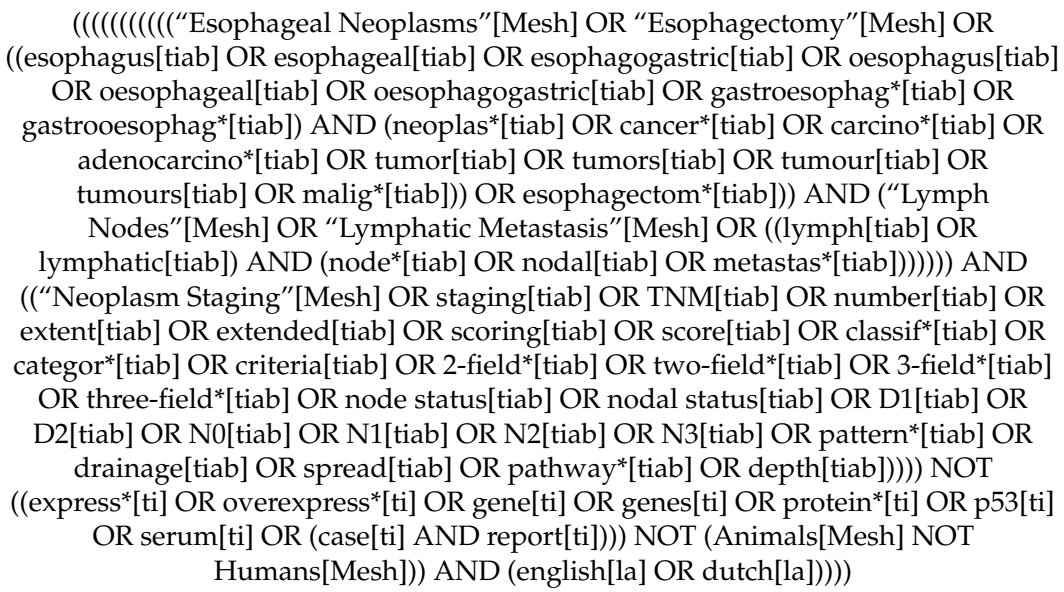 & 4106 \\
\hline
\end{tabular}

[Mesh] = Medical subject headings; [tiab] = words in title OR abstract; [ti] = words in title; [la] = language.

Table A2. Search strategy for Embase.com (22 July 2019).

\begin{tabular}{|c|c|c|}
\hline$\#$ & Searches & Results \\
\hline 1 & $\begin{array}{c}\text { exp esophagus tumor/or esophagus resection/or esophagectom*.ti,ab,kw. or } \\
\text { ((esophagus or esophageal or esophagogastric or oesophagus or oesophageal or } \\
\text { oesophagogastric or gastroesophag* or gastrooesophag* }{ }^{*} \text { and (neoplas* or } \\
\text { cancer }^{*} \text { or carcino* or adenocarcino* or tumor or tumors or tumour or tumours } \\
\text { or malig*)).ti,ab,kw. }\end{array}$ & 127708 \\
\hline 2 & $\begin{array}{c}\text { (exp lymph node/or exp lymph node metastasis/or ((lymph or lymphatic) and } \\
\left(\text { node }^{*} \text { or nodal or metastas }\right) \text { ).ti,ab,kw.) and (cancer staging/or (staging or TNM } \\
\text { or number or extent or extended or scoring or score or classif* or categor* or } \\
\text { criteria or } 2 \text {-field }{ }^{*} \text { or two-field* or } 3 \text {-field* or three-field }{ }^{*} \text { or node status or nodal } \\
\text { status or D1 or D2 or N0 or N1 or N2 or N3 or pattern* or drainage or spread or } \\
\text { pathway* or depth).ti,ab,kw.) }\end{array}$ & 208651 \\
\hline
\end{tabular}


Table A2. Cont.

\begin{tabular}{ccc}
\hline$\#$ & Searches & Results \\
\hline 3 & 1 and 2 & 10765 \\
\hline 4 & $\begin{array}{c}\text { (express* or overexpress* or gene or genes or protein* or p53 or serum or (case } \\
\text { and report)).ti. }\end{array}$ & 2592018 \\
\hline 5 & animal/ not human/ & 1423187 \\
\hline 6 & conference abstract.pt. or conference paper/ or letter/ & 4893909 \\
\hline 7 & 3 not 4 not 5 not 6 & 6444 \\
\hline 8 & limit 7 to (dutch or english) & 5195 \\
\hline
\end{tabular}

exp = EMtree keyword with explosion; de = EMtree keyword without explosion; .ab,ti,kw = words in title OR abstract OR keyword; $. \mathrm{ti}=$ words in title; $. \mathrm{pt}=$ publication type.

\section{References}

1. Mariette, C.; Piessen, G.; Briez, N.; Triboulet, J.P. The Number of Metastatic Lymph Nodes and the Ratio Between Metastatic and Examined Lymph Nodes Are Independent Prognostic Factors in Esophageal Cancer Regardless of Neoadjuvant Chemoradiation or Lymphadenectomy Extent. Ann. Surg. 2008, 247, 365-371. [CrossRef] [PubMed]

2. O'Riordan, J.M.; Rowley, S.; Murphy, J.O.; Ravi, N.; Byrne, P.J.; Reynolds, J.V. Impact of solitary involved lymph node on outcome in localized cancer of the esophagus and esophagogastric junction. J. Gastrointest. Surg. 2007, 11, 493-499. [CrossRef] [PubMed]

3. Okholm, C.; Svendsen, L.B.; Achiam, M.P. Status and prognosis of lymph node metastasis in patients with cardia cancer-A systematic review. Surg. Oncol. 2014, 23, 140-146. [CrossRef]

4. Koenig, A.M.; Prenzel, K.L.; Bogoevski, D.; Yekebas, E.F.; Bubenheim, M.; Faithova, L.; Vashist, Y.K.; Gawad, K.A.; Baldus, S.E.; Schneider, P.M.; et al. Strong impact of micrometastatic tumor cell load in patients with esophageal carcinoma. Ann. Surg. Oncol. 2009, 16, 454-462. [CrossRef] [PubMed]

5. Smit, J.K.; Pultrum, B.B.; van Dullemen, H.M.; Van Dam, G.M.; Groen, H.; Plukker, J.T.M. Prognostic factors and patterns of recurrence in esophageal cancer assert arguments for extended two-field transthoracic esophagectomy. Am. J. Surg. 2010, 200, 446-453. [CrossRef]

6. Phillips, A.W.; Lagarde, S.M.; Navidi, M.; Disep, B.; Griffin, S.M. Impact of extent of lymphadenectomy on survival, post neoadjuvant chemotherapy and trans-thoracic esophagectomy. Ann Surg. 2016, 265, 750-756. [CrossRef]

7. Castoro, C.; Scarpa, M.; Cagol, M.; Ruol, A.; Cavallin, F.; Alfieri, R.; Zanchettin, G.; Rugge, M.; Ancona, E. Nodal Metastasis From Locally Advanced Esophageal Cancer: How Neoadjuvant Therapy Modifies Their Frequency and Distribution. Ann. Surg. Oncol. 2011, 18, 3743-3754. [CrossRef]

8. Sharma, D.; Thakur, A.; Toppo, S.; Chandrakar, S.K. Lymph node counts in indians in relation to lymphadenectomy for carcinoma of the oesophagus and stomach. Asian J. Surg. 2005, 28, 116-120. [CrossRef]

9. Mizutani, M.; Murakami, G.; Nawata, S.I.; Hitrai, I.; Kimura, W. Anatomy of right recurrent nerve node: Why does early metastasis of esophageal cancer occur in it? Surg. Radiol. Anat. 2006, 28, 333-338. [CrossRef]

10. Prenzel, K.L.; Bollschweiler, E.; Schröder, W.; Mönig, S.P.; Drebber, U.; Vallboehmer, D.; Hölscher, A.H. Prognostic relevance of skip metastases in esophageal cancer. Ann. Thorac. Surg. 2010, 90, 1662-1667. [CrossRef]

11. Liu, J.; Liu, Q.; Wang, Y.; Xia, Z.; Zhao, G. Nodal skip metastasis is associated with a relatively poor prognosis in thoracic esophageal squamous cell carcinoma. Eur. J. Surg. Oncol. 2016, 42, 1202-1205. [CrossRef] [PubMed]

12. Akutsu, Y.; Matsubara, H. Lymph node dissection for esophageal cancer. Gen. Thorac. Cardiovasc. Surg. 2013, 61,397-401. [CrossRef] [PubMed]

13. Mariette, C.; Piessen, G. Oesophageal cancer: How radical should surgery be? Eur. J. Surg. Oncol. 2012, 38, 210-213. [CrossRef]

14. Japanese Esophageal Sociey. Japanese Classification of Esophageal Cancer, 11th Edition: Part I. Esophagus 2017, 14, 1-36. [CrossRef] [PubMed] 
15. Rice, T.W.; Patil, D.T.; Blackstone, E.H. AJCC/UICC staging of cancers of the esophagus and esophagogastric junction: Application to clinical practice. Ann. Cardiothorac. Surg. 2017, 6, 119-130. [CrossRef] [PubMed]

16. Ye, T.; Sun, Y.; Zhang, Y.; Zhang, Y.; Chen, H. Three-field or two-field resection for thoracic esophageal cancer: A meta-analysis. Ann. Thorac. Surg. 2013, 96, 1933-1941. [CrossRef]

17. van Rijswijk, A.S.; Hagens, E.R.C.; van der Peet, D.L.; van Berge Henegouwen, M.I.; Gisbertz, S.S. Differences in Esophageal Cancer Surgery in Terms of Surgical Approach and Extent of Lymphadenectomy: Findings of an International Survey. Ann. Surg. Oncol. 2019, 26, 2063-2072. [CrossRef]

18. Willaert, W.; Mareel, M.; Van De Putte, D.; Van Nieuwenhove, Y.; Pattyn, P.; Ceelen, W. Lymphatic spread, nodal count and the extent of lymphadenectomy in cancer of the colon. Cancer Treat. Rev. 2014, 40, 405-413. [CrossRef]

19. Salhab, M.; Patani, N.; Mokbel, K. Sentinel lymph node micrometastasis in human breast cancer: An update. Surg. Oncol. 2011, 20, e195-e206. [CrossRef]

20. Nappo, G.; Perinel, J.; El Bechwaty, M.; Adham, M. The standardization of pancreatoduodenectomy where are we? Pancreas 2016, 45, 493-502. [CrossRef]

21. Slim, K.; Nini, E.; Forestier, D.; Kwiatkowski, F.; Panis, Y.; Chipponi, J. Methodological index for non-randomized studies (Minors): Development and validation of a new instrument. ANZ J. Surg. 2003, 73, 712-716. [CrossRef]

22. Sharma, S.; Fujita, H.; Yamana, H.; Kakegawa, T. Patterns of lymph node metastasis in 3-field dissection for carcinoma in the thoracic esophagus. Surg. Today 1994, 24, 410-414. [CrossRef] [PubMed]

23. Van De Ven, C.; De Leyn, P.; Coosemans, W.; Van Raemdonck, D.; Lerut, T. Three-field lymphadenectomy and pattern of lymph node spread in T3 adenocarcinoma of the distal esophagus and the gastro-esophageal junction. Eur. J. Cardio Thorac. Surg. 1999, 15, 769-773. [CrossRef]

24. Igaki, H.; Kato, H.; Tachimori, Y.; Sato, H. Prognostic evaluation for squamous cell carcinomas of the lower thoracic esophagus treated with three-field lymph node dissection. Eur. J. Cardiothorac. Surg. 2001, 19, 887-893. [CrossRef]

25. Dresner, S.M.; Lamb, P.J.; Bennett, M.K.; Hayes, N.; Griffin, S.M. The pattern of metastatic lymph node dissemination from adenocarcinoma of the esophagogastric junction. Surgery 2001, 129, 103-109. [CrossRef]

26. Chen, J.; Liu, S.; Pan, J.; Zheng, X.; Zhu, K.; Zhu, J.; Xiao, J.; Ying, M. The pattern and prevalence of lymphatic spread in thoracic oesophageal squamous cell carcinoma. Eur. J. Cardiothorac. Surg. 2009, 36, 480-486. [CrossRef] [PubMed]

27. Tachimori, Y.; Nagai, Y.; Kanamori, N.; Hokamura, N.; Igaki, H. Pattern of lymph node metastases of esophageal squamous cell carcinoma based on the anatomical lymphatic drainage system. Dis. Esophagus 2011, 24, 33-38. [CrossRef] [PubMed]

28. Li, H.; Yang, S.; Zhang, Y.; Xiang, J.; Chen, H. Thoracic recurrent laryngeal lymph node metastases predict cervical node metastases and benefit from three-field dissection in selected patients with thoracic esophageal squamous cell carcinoma. J. Surg. Oncol. 2012, 105, 548-552. [CrossRef] [PubMed]

29. Kosugi, S.I.; Kawaguchi, Y.; Kanda, T.; Ishikawa, T.; Sakamoto, K.; Akaike, H.; Fujii, H.; Wakai, T. Cervical lymph node dissection for clinically submucosal carcinoma of the thoracic esophagus. Ann. Surg. Oncol. 2013, 20, 4016-4021. [CrossRef]

30. Cheng, J.; Kong, L.; Huang, W.; Li, B.; Li, H.; Wang, Z.; Zhang, J.; Zhou, T.; Sun, H. Explore the radiotherapeutic clinical target volume delineation for thoracic esophageal squamous cell carcinoma from the pattern of lymphatic metastases. J. Thorac. Oncol. 2013, 8, 359-365. [CrossRef]

31. Lin, Z.; Chen, W.; Chen, Y.; Peng, X.; Zhu, K.; Lin, Y.; Lin, Q.; Hu, Z. A new classification of lymph node metastases according to the lymph node stations for predicting prognosis in surgical patients with esophageal squamous cell carcinoma. Oncotarget 2016, 7, 76261-76273. [CrossRef] [PubMed]

32. Dong, Y.; Guan, H.; Huang, W.; Zhang, Z.; Zhao, D.; Liu, Y.; Zhou, T.; Li, B. Precise delineation of clinical target volume for crossing-segments thoracic esophageal squamous cell carcinoma based on the pattern of lymph node metastases. J. Thorac. Dis. 2015, 7, 2313-2320. [CrossRef] [PubMed]

33. Duan, X.-F.; Tang, P.; Shang, X.-B.; Jiang, H.-J.; Yu, Z.-T. The prevalence of lymph node metastasis for pathological T1 esophageal cancer: A retrospective study of 143 cases. Surg. Oncol. 2018, 27, 1-6. [CrossRef]

34. Park, S.Y.; Suh, J.W.; Kim, D.J.; Park, J.C.; Kim, E.H.; Lee, C.Y.; Lee, J.G.; Paik, H.C.; Chung, K.Y. Near-Infrared Lymphatic Mapping of the Recurrent Laryngeal Nerve Nodes in T1 Esophageal Cancer. Ann. Thorac. Surg. 2018, 105, 1613-1620. [CrossRef] [PubMed] 
35. Pedrazzani, C.; de Manzoni, G.; Marrelli, D.; Giacopuzzi, S.; Corso, G.; Minicozzi, A.M.; Rampone, B.; Roviello, F. Lymph node involvement in advanced gastroesophageal junction adenocarcinoma. J. Thorac. Cardiovasc. Surg. 2007, 134, 378-385. [CrossRef]

36. Gertler, R.; Stein, H.J.; Schuster, T.; Rondak, I.-C.; Hofler, H.; Feith, M. Prevalence and topography of lymph node metastases in early esophageal and gastric cancer. Ann. Surg. 2014, 259, 96-101. [CrossRef]

37. Künzli, H.T.; van Berge Henegouwen, M.I.; Gisbertz, S.S.; van Esser, S.; Meijer, S.L.; Bennink, R.J.; Wiezer, M.J.; Seldenrijk, C.A.; Bergman, J.J.G.H.M.; Weusten, B.L.A.M.; et al. Pilot-study on the feasibility of sentinel node navigation surgery in combination with thoracolaparoscopic lymphadenectomy without esophagectomy in early esophageal adenocarcinoma patients. Dis. Esophagus 2017, 30, 1-8. [CrossRef]

38. Management, C. Anatomy of lymphatic drainage of the esophagus and lymph node metastasis of thoracic esophageal cancer. Cancer Manag. Res. 2018, 10, 6295-6303.

39. Murakami, G.; Sato, I.; Shimada, K.; Dong, C.; Kato, Y.; Imazeki, T. Direct lymphatic drainage from the esophagus into the thoracic duct. Surg. Radiol. Anat. 1994, 16, 399-407. [CrossRef]

40. Akiyama, H.; Tsurumaru, M.; Udagawa, H.; Kajiyama, Y. Radical lymph node dissection for cancer of the thoracic esophagus. Ann. Surg. 1994, 220, 363-364. [CrossRef]

41. Wang, F.; Zheng, Y.; Wang, Z.; Zheng, Q.; Huang, Q.; Liu, S. Nodal Skip Metastasis in Esophageal Squamous Cell Carcinoma Patients Undergoing Three-Field Lymphadenectomy. Ann. Thorac. Surg. 2017, 104,1187-1193. [CrossRef] [PubMed]

42. He, S.-L.; Yang, Y.-S.; Wang, W.-P.; Zhang, H.-L.; Wang, Y.-C.; Chen, L.-Q. Prognostic Evaluation of Nodal Skip Metastasis for Thoracic Esophageal Squamous Cell Carcinoma. Ann. Thorac. Surg. 2019, 108, 1717-1723. [CrossRef] [PubMed]

43. Kumakura, Y.; Yokobori, T.; Yoshida, T.; Hara, K.; Sakai, M.; Sohda, M.; Miyazaki, T.; Yokoo, H.; Handa, T.; Yorifuji, H.; et al. Elucidation of the Anatomical Mechanism of Nodal Skip Metastasis in Superficial Thoracic Esophageal Squamous Cell Carcinoma. Ann. Surg. Oncol. 2018, 25, 1221-1228. [CrossRef] [PubMed]

44. Shin, S.; Kim, H.K.; Choi, Y.S.; Kim, K.; Shim, Y.M. Clinical stage T1-T2N0M0 oesophageal cancer: Accuracy of clinical staging and predictive factors for lymph node metastasis. Eur. J. Cardiothorac. Surg. 2014, 46, 274-279. [CrossRef] [PubMed]

45. Talsma, A.K.; Shapiro, J.; Looman, C.W.; van Hagen, P.; Steyerberg, E.W.; van der Gaast, A.; van Berge Henegouwen, M.I.; Wijnhoven, B.P.L.; van Lanschot, J.J.B.; CROSS Study Group. Lymph node retrieval during esophagectomy with and without neoadjuvant chemoradiotherapy: Prognostic and therapeutic impact on survival. Ann. Surg. 2014, 260, 783-786. [CrossRef] [PubMed]

46. Hagens, E.R.C.; Künzli, H.T.; van Rijswijk, A.S.; Meijer, S.L.; Mijnals, R.C.D.; Weusten, B.L.; Geijsen, E.D.; van Laarhoven, H.W.M.; van Berge Henegouwen, M.I.; Gisbertz, S.S. Distribution of lymph node metastases in esophageal adenocarcinoma after neoadjuvant chemoradiation therapy: A prospective study. Surg. Endosc. 2019. [CrossRef]

47. Ma, J.B.; Song, Y.P.; Yu, J.M.; Zhou, W.; Cheng, E.C.; Zhang, X.Q.; Kong, L. Feasibility of involved-field conformal radiotherapy for cervical and upper-thoracic esophageal cancer. Oncol. Res. Treat. 2011, 34, 599-604. [CrossRef]

48. Huang, W.; Li, B.; Gong, H.; Yu, J.; Sun, H.; Zhou, T.; Zhang, Z.; Liu, X. Pattern of lymph node metastases and its implication in radiotherapeutic clinical target volume in patients with thoracic esophageal squamous cell carcinoma: A report of 1077 cases. Radiother. Oncol. 2010, 95, 229-233. [CrossRef]

49. Zhang, J.; Liu, Y.; Che, F.; Luo, Y.; Huang, W.; Heng, X.; Li, B. Pattern of lymph node metastasis in thoracic esophageal squamous cell carcinoma with poor differentiation. Mol. Clin. Oncol. 2018, 8, 760-766. [CrossRef]

50. Hagens, E.R.C.; van Berge Henegouwen, M.I.; Van Sandick, J.W.; Cuesta, M.A.; Van Der Peet, D.L.; Heisterkamp, J.; Nieuwenhuijzen, G.A.P.; Rosman, C.; Scheepers, J.J.G.; Van Hillegersberg, R.; et al. Distribution of lymph node metastases in esophageal carcinoma [TIGER study]: Study protocol of a multinational observational study. BMC Cancer 2019, 19, 662. [CrossRef]

(C) 2020 by the authors. Licensee MDPI, Basel, Switzerland. This article is an open access article distributed under the terms and conditions of the Creative Commons Attribution (CC BY) license (http://creativecommons.org/licenses/by/4.0/). 